IOS Press

\title{
Dynamic fixation using rigid tape in rehabilitation after surgery of terrible triad injury of the elbow: A randomized trial
}

\author{
Shenxing Du ${ }^{\mathrm{a}}$, Lihong Wei ${ }^{\mathrm{b}}$, Bangjian $\mathrm{He}^{\mathrm{c}}$, Zhen Fang ${ }^{\mathrm{d}}$, Eryuan Zhou ${ }^{\mathrm{d}}$, Xiaoxiao $\mathrm{Ma}^{\mathrm{a}}$ and $\mathrm{Ju} \mathrm{Li}^{\mathrm{c}, *}$ \\ a Department of Rehabilitation, Dongyang People's Hospital, Dongyang, Zhejiang, China \\ ${ }^{\mathrm{b}}$ Department of Orthopedics, Dongyang People's Hospital, Dongyang, Zhejiang, China \\ ${ }^{\mathrm{c}}$ Department of Orthopedic Surgery, The First Affiliated Hospital of Zhejiang Chinese Medical University, \\ Hangzhou, Zhejiang, China \\ ${ }^{\mathrm{d}}$ Department of Rehabilitation, The Third Affiliated Hospital of Zhejiang Chinese Medical University, Hangzhou, \\ Zhejiang, China
}

Received 19 September 2020

Accepted 11 January 2021

\begin{abstract}
.
OBJECTIVE: The aim of the study was to identify the efficacy of dynamic fixation using rigid tape (RT) in rehabilitation after surgery of terrible triad injury of the elbow (TTIE).

METHODS: Sixty patients who underwent surgery of TTIE were equally randomly divided into RT group and hinged external fixation brace (HEFB) group. Dynamic fixations were applied for 8 weeks. General rehabilitation programs were performed for 3 months, 5 times a week. Follow-up (FU) was at six months. Main outcomes included pain (Visual Analogue Scale, VAS), muscle strength, range of motion (ROM), Elbow Function (Mayo Elbow Performance Index, MEPI), Quality of Life (QOL) (Short Form 36 Questionnaire, SF-36).

RESULTS: There were significant time $\mathrm{x}$ group interactions for pain, ROM, MEPI, SF-36 (all $p=0.000$ ), which demonstrated positive efficacy of both the two interventions. Difference at each time-point (except for baseline) of pain and ROM between the two groups was statistically significant (all $p<0.05$ ). Some differences between the two groups were not statistically significant which at $14 \mathrm{~d}$ on MEPI $(p=0.108)$ and at $21 \mathrm{~d}(p=0.259)$ and FU $(p=0.402)$ on QOL. Moreover, the increased muscle strength at each time-point had no statistically significant difference between the two groups (all $p>0.05$ ).

CONCLUSIONS: Both RT and HEFB could significantly improve the postoperative functional outcomes of the TTIE. However, early rehabilitation intervention could increase pain, which affected the corresponding function (MEPI) and QOL. Note that this kind of impact was short-term and reversible. The muscle strength and ROM were not affected by the increased severe pain, maintaining a trend of improvement. In addition, the subjects in the RT group improved faster and more efficiently and had better results with pain, ROM, MEPI, and QOL compared to the subjects in the HEFB group.
\end{abstract}

Keywords: Orthopedic fixation devices, athletic tape, braces, rehabilitation, elbow

${ }^{*}$ Corresponding author: Ju Li, Department of Orthopedic Surgery,
the First Affiliated Hospital of Zhejiang Chinese Medical University,
Youdian Road, No. 54, Shangcheng District, Hangzhou, Zhejiang,
310006, China. Tel.: +86 13735807172; E-mail: 48490441@qq.com.

\section{Introduction}

Terrible triad injury of the elbow (TTIE), which was named by Hotchkiss [1-4], describes a condition on dislocation of the elbow with fractures of the radial head and ulnar coronoid process $[1-3,5]$. The elbow is 
the second most dislocated joint in the body [3,6-8], $18 \%-45 \%$ would be TTIE $[2,3]$. This type of injury occurs during falls onto an outstretched hand, falls from a height, motor vehicle accidents, or other high-energy trauma [9]. The mechanism is hyperextension and valgus stress applied to the pronated forearm [5,9]. This injury often causes instability of the elbow which requires surgical intervention. The clinical efficacy of this compound injury is not satisfactory, often associated with complications, such as stiffness, pain, instability, traumatic arthritis and heterotopic ossification [2]. The primary purpose of surgery is to stabilize the elbow and ensure the early movement to avoid stiffness.

However, surgery may not provide sufficient stability for the elbow joint. Therefore, braces, such as hinged external fixation brace (HEFB), are used to supplement this deficiency to ensure the early postoperative mobility training. Because fixators must maintain the stability of the joints during the healing of soft tissues and bone injuries, it often lags in rehabilitation interventions [2]. Complications include instability, stiffness, infection, pain, ulnar neuropathy, malunion, nonunion, heterotopic ossification arthrosis, osteoarthritis and contracture [10-14]. Re-operation is necessary in as many as $28 \%$ of patients $[10,13,15]$.

As far as the current literature is concerned, there is no evidence to reveal the optimal postoperative training intervention time point. In particular, it is rare to start rehabilitation interventions 1 to 2 weeks after surgery $[16,17]$. Instability is thought to be more common in smaller avulsion-type coronoid fracture, possibly due to the more challenging fixation [10]. Therefore, most rehabilitation practitioners prefer to intervene after the joint is stabilized to prevent the occurrence of cubitus varus or valgus deformity. The joint stiffness occurs which increases the difficulty of recovery and the patient's pain, especially for the functional recovery of a complex joint like the elbow where stiffness is poorly tolerated and instability is devastating [5].

Rigid tape (RT) is a favorite in the field of rehabilitation medicine, especially it can stabilize joints, and can even change the direction of joint movement in the direction we need to promote muscles to complete their role as dynamic fixators. But it also has limitations, such as the high requirements for surgical wound healing.

Therefore, our main objectives were to compare results of two types of fixation. We hypothesized that dynamic fixation by using RT applied on TTIE would promote functional recovery more effectively than HEFB. Furthermore, RT would not cause serious adverse events (AEs) and rehabilitation interventions initiated since 2-4 weeks after surgery would be safe.

\section{Methods}

\subsection{Study design}

This study was conducted between July 2017 and June 2019. This was a single-blinded randomized controlled study. Sixty patients were equally divided into the RT group and the HEFB group by a random digital table with each card placed in a brown opaque envelope. The random plan is only known to the project leader. According to the PEDro Scale [18], the physiotherapist who evaluated all the outcomes was blinded (singleblind: evaluator). The study was approved by the Ethics Committee of the First Affiliated Hospital of Zhejiang Chinese Medical University (No. 2017LCSY0010) and the patients provided informed consent.

\subsection{Patients}

Inclusion criteria: 2 to 4 weeks after surgery; surgical wounds healing well, dry and without exudation; stable vital signs. Exclusion criteria: accompanied by other muscle, bone, and nerve injuries; osteoporosis; severe heart, liver, and kidney diseases; severe diabetes and other organic diseases of the endocrine system.

\subsection{Treatments}

RT group: According to the inter-bone position of each joint (humeroulnar joint, humeroradial joint, and proximal radioulnar joint), use RT to recover the boneto-bone dynamic position relationship (re-tape every day). Meanwhile, instruct the patients to feel the direction of the RT pulling force, so as to use the muscle strength accordingly. In order to meet the dynamic position relationship, we applied the RT for 1) stable dynamic supination, even though the supination is regarded as the least stable position in the conventional sense, 2) dynamically strengthening lateral side of the elbow (Fig. 1A and B). The patients were also asked to do some fit-in exercises, such as bouncing a ball off ground or wall. HEFB group: The patients were asked to wear HEFBs (Fig. 1C and D) for 24 hours a day and perform no or slight painful activities (even activities that involved pronation and supination) during the off-treatment phase. Dynamic fixations were applied for 8 weeks. General rehabilitation programs, such as strength training (10 minutes), range of motion training (10 minutes), activities of daily living (ADLs) training (20 minutes), and modalities (ultrasound: 10 minutes, neuromuscular electrical stimulation: 20 minutes, transcutaneous electrical nerve stimulation: 20 minutes), were performed by all the patients and conducted by physiotherapists for 3 months, 5 times a week. 
Table 1

Characteristics of the 60 patients in terms of age, gender, affected side and PORT

\begin{tabular}{lllrrc}
\hline & RT group $(n=30)$ & HEFB group $(n=30)$ & $t / \chi^{2}$ & $p$ (sig) & 95\% confidence interval $(\mathrm{CI})$ \\
\hline Age (yr) & $48.70 \pm 14.61$ & $46.80 \pm 13.80$ & 0.518 & 0.607 & $-5.447-9.247$ \\
Gender (male/female) & $20 / 10$ & $17 / 13$ & 0.635 & 0.426 & \\
Side (left/right) & $18 / 12$ & $13 / 17$ & 1.669 & 0.196 & $-2.200-1.933$ \\
PORT (d) & $23.20 \pm 3.78$ & $23.33 \pm 4.20$ & -0.129 & 0.898 & \\
\hline
\end{tabular}
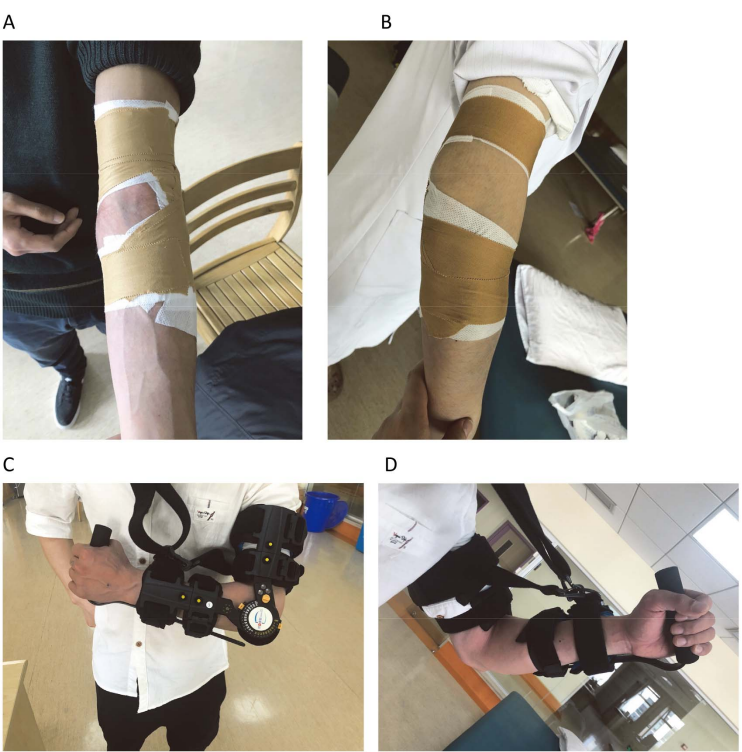

Fig. 1. A) and B) Patients who applied RT. C) and D) Patients who applied HEFB.

\subsection{Assessments}

Pain: Measure pain during activity. Visual Analogue Scale (VAS). Draw a $10 \mathrm{~cm}$ horizontal line on the paper, one end of the horizontal line is 0 , indicating no pain; the other end is 10 , indicating severe pain. Ask the patient to draw a mark on the horizontal line according to their feelings to indicate the degree of pain. Muscle Strength: Based on biceps brachii. Manual Muscle Testing (MMT). Grade 0: No evidence of muscle contractility; Grade 1: Evidence of slight contractility with no evidence of joint motion even with gravity eliminated; Grade 2: Complete range of motion (ROM) with gravity eliminated; Grade 3: Complete ROM against gravity with no resistance; Grade 4: Complete ROM against with some resistance; Grade 5: Complete ROM against gravity with full resistance. ROM: With the forearm in active full supination. Use goniometer. Center fulcrum over the lateral epicondyle of the humerus. Align proximal arm with the lateral mid-line of the humerus, using the center of the arcomion process for reference. Align the distal arm with the lateral mid-line of the radius, using the radial styliod process for reference. We define this ROM as the difference between the biggest angle and the smallest angle of the elbow flexion, i.e. the arc of flexion. Elbow Function: Mayo Elbow Performance Index (MEPI) [19]. It consists of pain (45 points), ROM (20 points), stability (10 points) and ADL (25 points). Quality of Life (QOL): Short Form 36 Questionnaire (SF-36) [20]. It includes general health, pain, activities, and physical and emotional health problems. All the assessments mentioned above were measured before rehabilitation intervention, and 7 days, 14 days, 21 days, 1 month, and 3 months after the beginning, as well as 6-month follow-up (FU). Safety: Safety was assessed via AE reporting, physical examinations, vital sign measurement, $\mathrm{x}$-ray, etc. during the process of this study when needed.

\subsection{Statistical analysis}

Statistics were conducted in IBM SPSS Statistics Version for Mac 23.0 and significance was set at $p>$ 0.05 . Differences in age, postoperative rehabilitation time (PORT), pain, ROM, MEPI, and QOL were assessed using repeated measures models and multivariate analysis of variance (MANOVA). Differences in gender, affected side, and muscle strength were assessed using Chi-square $\left(\chi^{2}\right)$ tests. Differences in muscle strength were assessed using Fisher's Exact Test.

\section{Results}

Table 1 shows the characteristics of the 60 patients in terms of age, gender, affected side and PORT for which there were no statistically significant differences $(p>0.05)$ between the two groups. There were no statistically significant differences $(p>0.05)$ between the two groups during the initial measurement for all variables (baseline) (Tables 2 and 3 ).

There were significant time $\mathrm{x}$ group interactions for pain, ROM, MEPI, SF-36 (all $p=0.000$ ), which demonstrated positive efficacy of both the two interventions. Difference at each time-point (except for baseline) of pain and ROM between the two groups was statistically significant (Figs 2 and 3). Some differences 
Table 2

Pain, ROM, MEPI, SF-36. Values are Means \pm SD

\begin{tabular}{|c|c|c|c|c|c|c|c|c|}
\hline & & Baseline & $7 \mathrm{~d}$ & $14 \mathrm{~d}$ & $21 \mathrm{~d}$ & $1 \mathrm{~m}$ & $3 \mathrm{~m}$ & Follow-up \\
\hline RT group & Pain & $3.23 \pm 1.25$ & $6.30 \pm 1.18$ & $6.33 \pm 1.09$ & $3.57 \pm 0.73$ & $2.10 \pm 0.80$ & $1.20 \pm 0.85$ & $0.50 \pm 0.57$ \\
\hline \multirow[t]{3}{*}{$(n=30)$} & ROM & $6.63 \pm 3.24$ & $22.97 \pm 5.17$ & $50.00 \pm 6.32$ & $69.17 \pm 7.39$ & $82.90 \pm 7.60$ & $108 \pm 7.07$ & $109.90 \pm 6.27$ \\
\hline & MEPI & $30.00 \pm 8.51$ & $17.5 \pm 10.15$ & $24.33 \pm 10.48$ & $59.33 \pm 12.78$ & $73.17 \pm 6.09$ & $84.67 \pm 7.87$ & $91.50 \pm 9.48$ \\
\hline & SF-36 & $81.43 \pm 5.47$ & $73.47 \pm 4.88$ & $74.27 \pm 5.32$ & $92.07 \pm 5.39$ & $107.50 \pm 4.49$ & $120.67 \pm 5.64$ & $130.63 \pm 5.82$ \\
\hline HEFB & Pain & $3.03 \pm 1.45$ & $4.50 \pm 1.31$ & $4.60 \pm 1.28$ & $5.00 \pm 1.23$ & $5.10 \pm 1.12$ & $3.33 \pm 1.06$ & $1.03 \pm 0.85$ \\
\hline group & ROM & $6.03 \pm 1.92$ & $13.87 \pm 4.13$ & $25.50 \pm 8.03$ & $42.77 \pm 8.56$ & $53.9 \pm 7.25$ & $79.00 \pm 10.18$ & $80.10 \pm 9.97$ \\
\hline \multirow[t]{2}{*}{$(n=30)$} & MEPI & $30.50 \pm 9.86$ & $31.17 \pm 14.84$ & $29.33 \pm 13.11$ & $32.33 \pm 14.13$ & $41.33 \pm 14.14$ & $61.33 \pm 9.91$ & $80.33 \pm 9.73$ \\
\hline & SF-36 & $82.07 \pm 4.96$ & $79.70 \pm 4.43$ & $81.10 \pm 4.42$ & $90.23 \pm 6.97$ & $103.73 \pm 8.01$ & $113.10 \pm 6.10$ & $129.10 \pm 8.06$ \\
\hline$P$ (sig) & Pain & 0.569 & 0.000 & 0.000 & 0.000 & 0.000 & 0.000 & 0.006 \\
\hline \multirow[t]{7}{*}{$95 \% \mathrm{CI}$} & & $2.783-3.4831$ & $5.078-5.722$ & $5.160-5.774$ & $4.022-4.545$ & $3.347-3.583$ & $2.019-2.515$ & $0.579-0.954$ \\
\hline & ROM & 0.387 & 0.000 & 0.000 & 0.000 & 0.000 & 0.000 & 0.000 \\
\hline & & $5.645-7.022$ & $17.207-19.626$ & $35.882-39.618$ & $53.900-58.033$ & $66.481-70.319$ & $91.236-95.764$ & 92.849-91.151 \\
\hline & MEPI & 0.834 & 0.000 & 0.108 & 0.000 & 0.000 & 0.000 & 0.000 \\
\hline & & $27.871-32.629$ & $21.048-27.618$ & $23.766-29.901$ & $42.352-49.314$ & $54.437-60.063$ & $70.688-75.312$ & $83.434-88.400$ \\
\hline & SF-36 & 0.640 & 0.000 & 0.000 & 0.259 & 0.028 & 0.000 & 0.402 \\
\hline & & $80.401-83.099$ & 75.379-77.788 & 76.419-78.948 & $89.541-92.759$ & $103.939-107.295$ & $115.365-128.050$ & $118.401-131.684$ \\
\hline
\end{tabular}

Table 3

Muscle strength: Number of patients who reported a specific grade of MMT

\begin{tabular}{lcccccccc}
\hline & Grade & Baseline & $7 \mathrm{~d}$ & $14 \mathrm{~d}$ & $21 \mathrm{~d}$ & $1 \mathrm{~m}$ & $3 \mathrm{~m}$ & Follow-up \\
\hline RT group $(n=30)$ & 2 & 3 & 0 & 0 & 0 & 0 & 0 & 0 \\
& 3 & 9 & 3 & 0 & 0 & 0 & 0 & 0 \\
& 4 & 16 & 15 & 6 & 2 & 0 & 0 & 0 \\
HEFB group $(n=30)$ & 5 & 2 & 12 & 24 & 28 & 30 & 30 & 30 \\
& 2 & 4 & 1 & 0 & 0 & 0 & 0 & 0 \\
& 3 & 10 & 6 & 3 & 1 & 0 & 0 & 0 \\
$\chi^{2}$ & 4 & 14 & 15 & 8 & 4 & 4 & 1 & 0 \\
$P$ (sig) & 5 & 2 & 8 & 19 & 25 & 26 & 29 & 30 \\
\hline
\end{tabular}

between the two groups were not statistically significant which at $14 \mathrm{~d}(p=0.108)$ on MEPI (Table 2, Fig. 4) and at $21 \mathrm{~d}(p=0.259)$ and FU $(p=0.402)$ on QOL (Table 2, Fig. 5). Moreover, the increased muscle strength at each time-point had no statistically significant difference between the two groups (all $p>0.05$ ) (Table 3).

\section{Discussion}

It is generally considered that early activities after TTIE are ambiguous. However, the results of this study show it could be safe for rehabilitation practitioners to intervene earlier.

Pain as an individual variable has a positive significance in the early rehabilitation, although it is involved in the MEPI and SF-36. It is worth noting that early activities caused the significantly increased pain that was more severe in the RT group and longer lasting in the HEFB group (Fig. 2). Consequently, the MEPI and SF-36 of the RT group dropped down at $7 d$ and $14 d$ and rose sharply at 21d (Figs 4 and 5). Fortunately, the strength (Table 3) and ROM (Fig. 3) were not affected. Although the severest pain occurred respectively in the first 14 days in the RT group and in the first 1 month in the HEFB group, each of them fell apace subsequently (Fig. 2). It indicates that the early pain due to activities is safe and reversible under both kinds of dynamic fixations. If only considering pain, we prefer RT as it can cause less lasting and severe pain.

The only variable which had no statistically significant differences between the two groups was the muscle strength (Table 3). It indicates that no matter what type of activities used to improve muscle strength, no differences in efficiency for dynamic fixations were found.

The most important and notable variable is the ROM. Seth and Thomas ${ }^{5}$ stated that a full range of flexion and extension was not necessary, and full extension may in fact lead to disruption of coronoid fracture or anterior capsular repairs. Assessing motion through the functional range from $30^{\circ}$ to $130^{\circ}$ of flexion suffices. Most practitioners [1,16,21-23] involved strive for greater and greater ROM. The solution we chose to get a handle on the subtle incongruence was the RT. The most common use of RT is to stabilize the joints. We modified 
Pain

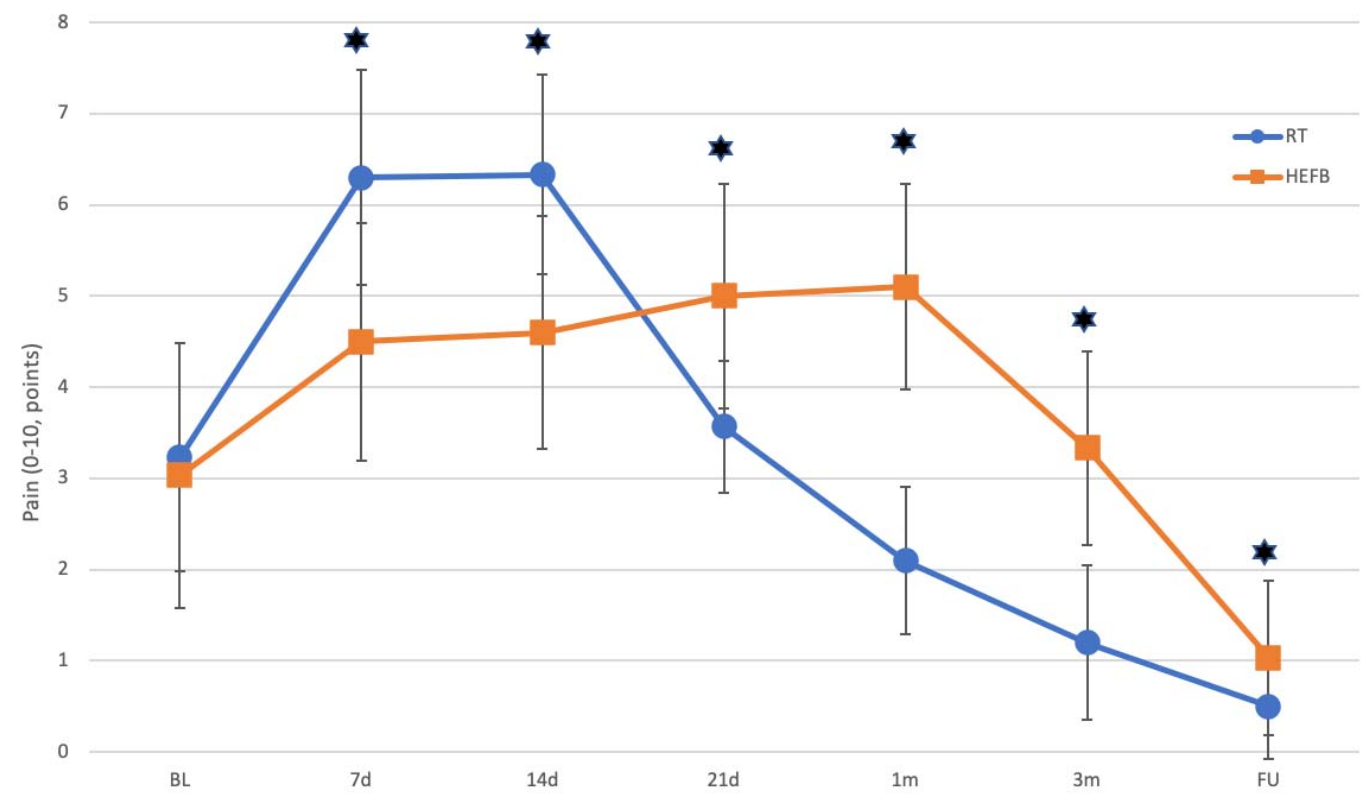

$-1$

Fig. 2. Pain (VAS; where $0=$ no pain and $10=$ worst imaginable pain). ${ }^{*}$ Denotes significantly different between the two groups at $p<0.05$.

ROM

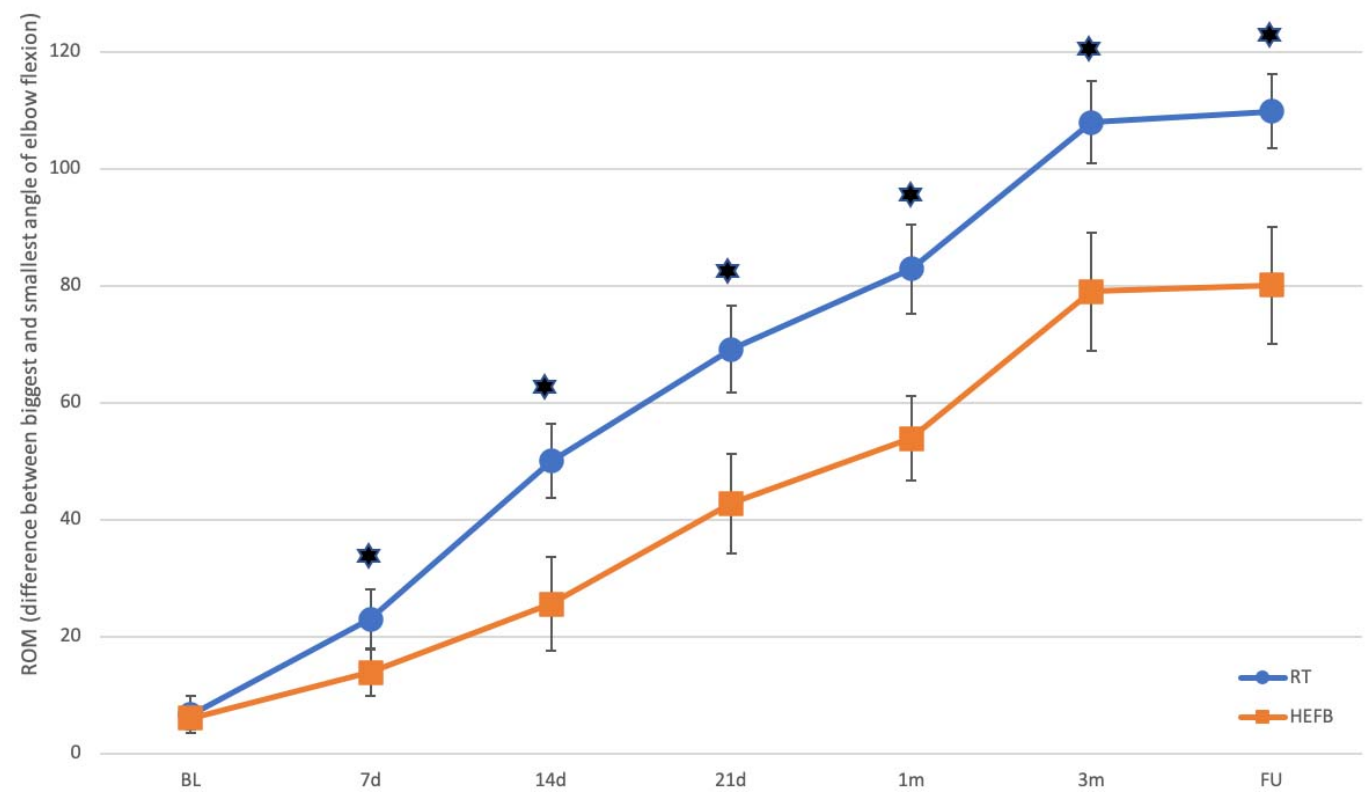

Fig. 3. ROM (difference between biggest and smallest angle of elbow flexion, i.e. arc of elbow flexion). ${ }^{*}$ Denotes significantly different between the two groups at $p<0.05$. 


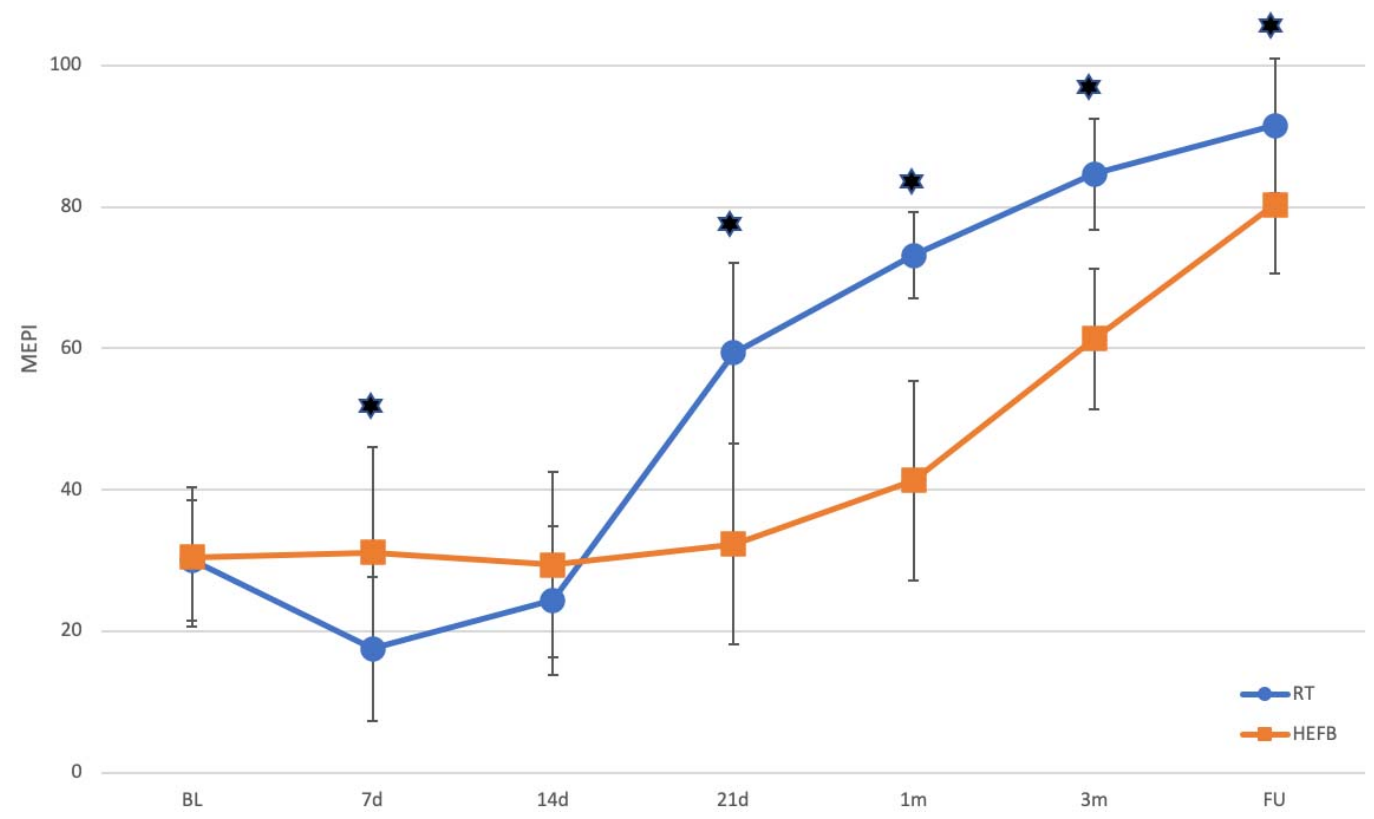

Fig. 4. Elbow Function (MEPI). ${ }^{*}$ Denotes significantly different between the two groups at $p<0.05$.

QOL (SF-36)

150

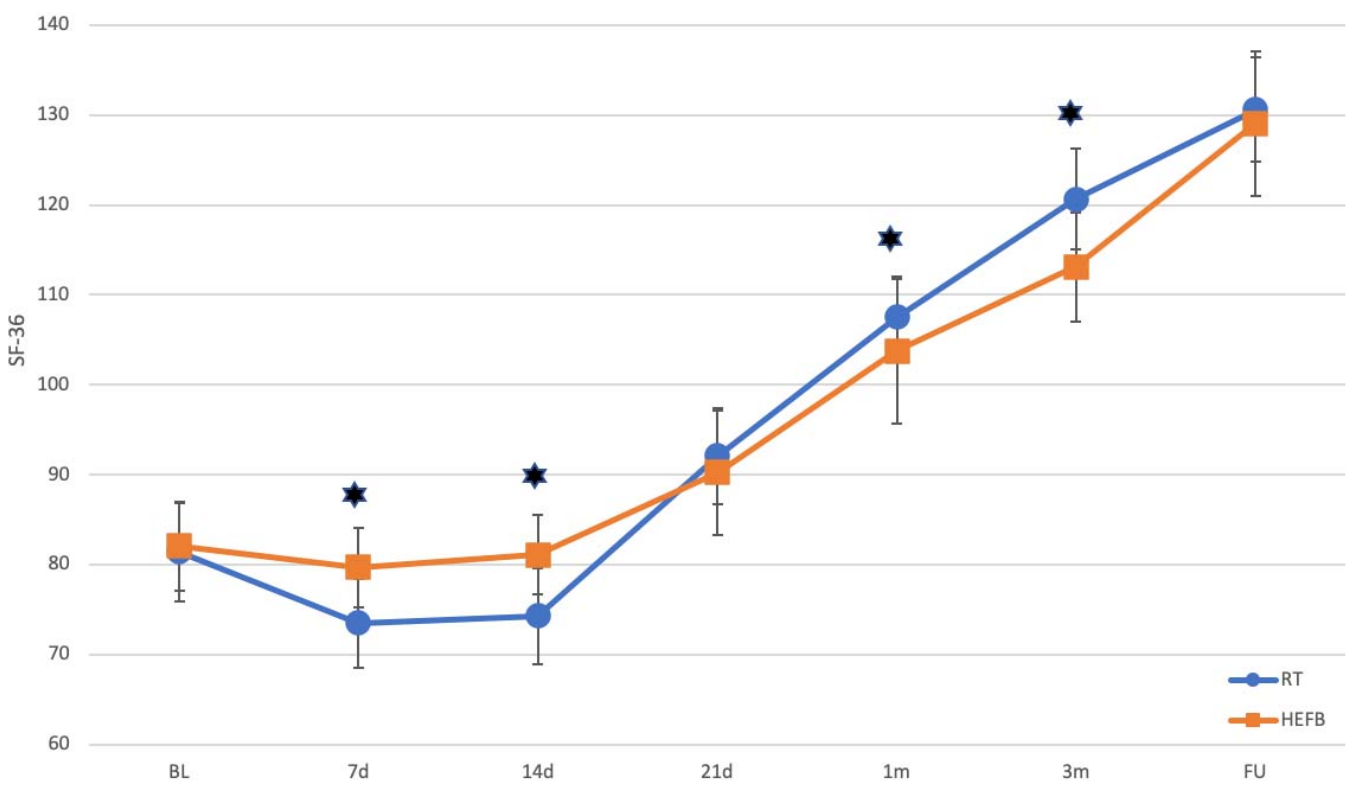

Fig. 5. QOL (SF-36 Questionnaire). ${ }^{*}$ Denotes significantly different between the two groups at $p<0.05$. 
the method of application based on the 3D dynamic joint technique for dynamic stabilization of humeroulnar joint, humeroradial joint and proximal radioulnar joint. Our results confirmed that the RT could more significantly improve the arc of elbow flexion than HEFB $(p<0.05)$ (Fig. 3). The final ROM (109.90 \pm 6.27$)$ of the RT group is greater than most of other studies $[3,5,9,11,24]$, however, applying HEFB (80.10 \pm $9.97)$ is lower than other studies $[3,5,9,11,23-25]$. The final data of other studies did not involve measuring at certain time points, they just measured the results whenever rehabilitation was finished, i.e. their results did not include the length of time for their rehabilitation, so we cannot comparatively analyze the differences.

With the improvement of ROM, the stability was also improved synchronously. As we mentioned above, the reason why the functional scores based on MEPI decreased in the early phrase was due to the increased severe pain. Our data shows (Fig. 4) that as the pain released, the MEPI of the RT group (14d-21d) increased more sharply than in the HEFB group (1m-3m). It indicates that the RT could prevent or decrease the occurrence of cubitus varus or valgus deformity more efficiently, as cubitus varus or valgus deformity is responsible for elbow stability. Moreover, the patients who applied RT could return to ADLs earlier. This may be because the patients were asked to feel the direction of the pull based on the applied RT while doing activities in order to rebuild the muscle memory for the subtle congruence. Compared to other studies [3,5,11,24], there is a greater final score $(91.50 \pm 9.48)$ in the MEPI of the RT group, whereas a similar one $(80.10 \pm 9.97)$ occurred in the HEFB group. Due to heterogeneity we mentioned above, comparative analysis is not available.

Similar to the MEPI, the QOL based on SF-36 was affected by the pain. However, its growth point occurred earlier than the MEPI (Fig. 5). This is because the functional recovery enhanced the patient's self-confidence.

Against dominancy and affected side, all of our patients were right-handed. The impact of dominancy is not necessary to discuss here, because we asked every patient to use their affected hand to perform most ADLs, such as washing their face, brushing their teeth, using chopsticks or spoons, and using their un-affected hand to do heavy work. The patients performed very well, even though they were like fishes out of water in the beginning.

Safety is the most important variable for rehabilitation. No malunion, nonunion or heterotopic ossification was found in our study. However, four patients in the RT group suffered slight skin allergy, and one suffered mild skin allergy. The skin allergy was a red, itchy rash, no blisters or bumps showed up. We would eliminate them, but they insisted on keeping going. Finally, we kept them in not only because of their will, but also because the allergy was cured by Ebastine Tablets (1 tablet a day), Loratadine Tablets (1 tablet a day), Prednisolone Acetate Tablets ( 1 tablet a time, 3 times a day) and Hydrocortisone Butyrate Cream (3 times a day), while there was no treatment discontinued. There is no direct evidence against applying RT when an allergy occurs. We considered whether the allergic reactions were due to glue. A case report written by Christoffers et al. [26] stated that unmodified colophonium as a well-known sensitizer which could be used in medical adhesives could cause allergic reactions in $0.7-8.0 \%$ of the patients.

Limitations of our study are the relatively short 3month intervention duration and 6-month FU, and the sample size of this study is not large. Allergy is unavoidable for the application of RT. Fortunately, it had little impact on the process of our study. In addition, the application of RT as we are using requires practitioners to take relevant professional trainings.

\section{Conclusions}

Overall, both RT and HEFB could significantly improve the postoperative functional outcomes of the TTIE. However, early rehabilitation intervention could increase pain, which affected the corresponding function (MEPI) and QOL. Note that this kind of impact was short-term and reversible. The muscle strength and ROM were not affected by the increased severe pain, maintaining a trend of improvement. In addition, the subjects in the RT group improved faster and more efficiently and had better results with pain, ROM, MEPI, and QOL compared to the subjects in the HEFB group.

\section{Conflict of interest}

No funding was received, and no conflicts of interest have been reported by the authors or by any individuals in control of the content of this article.

\section{References}

[1] Mathew PK, Athwal GS, King GJ. Terrible triad injury of the elbow: current concepts. J Am Acad Orthop Surg. 2009 Mar; 17(3): 137-51. doi: 10.5435/00124635-200903000-00003. 
[2] Hotchkiss RN. Fractures and dislocations of the elbow. Rockwood and Green's Fractures in Adults, Vol.1, 4th ed phyladelphia: J.B.Lippincott. 1996: 929-1024.

[3] Jiménez-Martín A, Contreras-Joya M, Navarro-Martínez S, Najarro-Cid FJ, Santos-Yubero FJ, Pérez-Hidalgo S. Clinical results of radial arthroplasty in Hotchkiss' terrible triad, a case series of 47. Rev Esp Cir Ortop Traumatol. Mar-Apr 2020; 64(2): 83-91. doi: 10.1016/j.recot.2019.11.003. Epub 2019 Dec 18.

[4] Hotchkiss RN, Weiland AJ. Valgus stability of the elbow. J Orthop Res. 1987; 5(3): 372-7. doi: 10.1002/jor.1100050309.

[5] Dodds SD, Fishler T. Terrible triad of the elbow. Orthop Clin North Am. 2013 Jan; 44(1): 47-58. doi: 10.1016/j.ocl.2012.08. 006.

[6] Fitzpatrick MJ, Diltz M, McGarry MH, Lee TQ. A new fracture model for "terrible triad" injuries of the elbow: influence of forearm rotation on injury patterns. J Orthop Trauma. 2012 Oct; 26(10): 591-6. doi: 10.1097/BOT.0b013e31824135af.

[7] Ozel O, Demircay E. Review of management of unstable elbow fractures. World J Orthop. 2016 Jan 18; 7(1): 50-4. doi: 10.5312/wjo.v7.i1.50.

[8] Kuhn MA, Ross G. Acute elbow dislocations. Orthop Clin North Am. 2008 Apr; 39(2): 155-61, v. doi: 10.1016/j.ocl. 2007.12.004.

[9] Kovacevic D, Vogel LA, Levine WN. Complex elbow instability: radial head and coronoid. Hand Clin. 2015 Nov; 31(4): 547-56. doi: 10.1016/j.hcl.2015.06.004. Epub 2015 Jul 26.

[10] Bohn K, Ipaktchi K, Livermore M, Cao J, Banegas R. Current treatment concepts for "terrible triad" injuries of the elbow. 2014 Dec; 37(12): 831-7. doi: 10.3928/01477447-2014112406.

[11] Chemama B, Bonnevialle N, Peter O, Mansat P, Bonnevialle P. Terrible triad injury of the elbow: how to improve outcomes? Orthop Traumatol Surg Res. 2010 Apr; 96(2): 147-54. doi: 10.1016/j.rcot.2010.02.008.

[12] Seijas R, Ares-Rodriguez O, Orellana A, Albareda D, Collado D, Llusa M. Terrible triad of the elbow. J Orthop Surg (Hong Kong). 2009 Dec; 17(3): 335-9. doi: 10.1177/23094990090 1700319 .

[13] Watters TS, Garrigues GE, Ring D, Ruch DS. Fixation versus replacement of radial head in terrible triad: is there a difference in elbow stability and prognosis? Clin Orthop Relat Res. 2014 Jul; 472(7): 2128-35. doi: 10.1007/s11999-013-3331-x.

[14] Rodriguez-Martin J, Pretell-Mazzini J, Andres-Esteban EM, Larrainzar-Garijo R. Outcomes after terrible triads of the elbow treated with the current surgical protocols. A review. Int Orthop. 2011 Jun; 35(6): 851-60. doi: 10.1007/s00264-0101024-6. Epub 2010 May 8.

[15] Garrigues GE, Wray WH 3rd, Lindenhovius ALC, Ring DC, Ruch DS. Fixation of the coronoid process in elbow fracturedislocations. J Bone Joint Surg Am. 2011 Oct 19; 93(20): 1873-81. doi: 10.2106/JBJS.I.01673.
[16] Pugh DMW, McKee MD. The "terrible triad" of the elbow. Tech Hand Up Extrem Surg. 2002 Mar; 6(1): 21-9. doi: 10. 1097/00130911-200203000-00005.

[17] Zeiders GJ, Patel MK. Management of unstable elbows following complex fracture-dislocations - the "terrible triad" injury. J Bone Joint Surg Am. 2008 Nov; 90(Suppl 4): 75-84. doi: 10.2106/JBJS.H.00893.

[18] Maher CG, Sherrington C, Herbert RD, Moseley AM, Elkins M. Reliability of the PEDro scale for rating quality of randomized controlled trials. Phys Ther. 2003 Aug; 83(8): 713-21.

[19] Gonçalves LBJ, Neto JAES, Filho MRCC, Andrade RP, Andrade MAP, Gomes AH, Vilela JCS. Terrible triad of the elbow: influence of radial head treatment. Rev Bras Ortop. 2014 Jul 11; 49(4): 328-33. doi: 10.1016/j.rboe.2014.07.001. eCollection Jul-Aug 2014.

[20] Lieshout EMMV, Iordens GIT, Polinder S, Eygendaal D, Verhofstad MHJ, Schep NWL, Hartog DD. Early mobilization versus plaster immobilization of simple elbow dislocations: a cost analysis of the FuncSiE multicenter randomized clinical trial. Arch Orthop Trauma Surg. 2020 Jul; 140(7): 877-886. doi: 10.1007/s00402-019-03309-1. Epub 2019 Nov 23.

[21] Pugh DMW, Wild LM, Schemitsch EH, King GJW, McKee MD. Standard surgical protocol to treat elbow dislocations with radial head and coronoid fractures. J Bone Joint Surg Am. 2004 Jun; 86(6): 1122-30. doi: 10.2106/00004623-20040600000002.

[22] O'Driscoll SW, Jupiter JB, Cohen MS, Ring D, McKee MD Difficult elbow fractures: pearls and pitfalls. Instr Course Lect. 2003; 52: 113-34. PMID: 12690844

[23] Forthman C, Henket M, Ring DC. Elbow dislocation with intra-articular fracture: the results of operative treatment without repair of the medial collateral ligament. J Hand Surg Am. 2007 Oct; 32(8): 1200-9. doi: 10.1016/j.jhsa.2007.06.019.

[24] Contreras-Joya M, Jiménez-Martín A, Santos-Yubero FJ, Navarro-Martínez S, Najarro-Cid FJ, Sánchez-Sotelo J, PérezHidalgo S. Radial head arthroplasty, 11 years experience: a series of 82 patients. Rev Esp Cir Ortop Traumatol. Sep-Oct 2015; 59(5): 307-17. doi: 10.1016/j.recot.2015.02.001. Epub 2015 Mar 30.

[25] Lindenhovius ALC, Jupiter JB, Ring D. Comparison of acute versus subacute treatment of terrible triad injuries of the elbow. J Hand Surg Am. Jul-Aug 2008; 33(6): 920-6. doi: 10.1016/j. jhsa.2008.02.007.

[26] Christoffers WA, Coenraads PJ, Schuttelaar ML. Bullous allergic reaction caused by colophonium in medical adhesives. Contact Dermatitis. 2014 Apr; 70(4): 256-7. doi: 10.1111/cod. 12170. 